\title{
Delivery and death on railway track: A case report
}

\author{
N. T. Satish ${ }^{1 \star}$, Anitha $\mathrm{S}^{2}, \mathrm{~S}$. Harish ${ }^{1}$ and Girish Chandra ${ }^{1}$ \\ ${ }^{1}$ Department of Forensic Medicine, MS Ramaiah Medical College, Bangalore, Karnataka, India. \\ ${ }^{2}$ Department of Forensic Medicine, Adichunchunagiri Medical College, BG Nagar, Mandya, India.
}

Accepted 3 July, 2013

\begin{abstract}
Injuries and deaths due to accidents are inescapable in the modern way of living. The accidental deaths are mostly due to the road traffic, but the deaths due to railway fatalities are also not negligible, especially in the areas where railway traffic is high. Southern railways are major mode of transportation of the passengers and goods; consequently, the incidences of railway fatalities and mishaps are also high in South India. Most of the reported cases of railway deaths led to the conclusion that either the victim was directly hit by the train or died due to some other reason, but the body was recovered in the vicinity of the railway platforms. Here, we are discussing the injuries in a young pregnant woman in a train and pedestrian accident, where the injury to the abdomen resulted into the expulsion of preterm fetus and death of pregnant woman.
\end{abstract}

Key words: Railway accident, death, delivery, autopsy.

\section{INTRODUCTION}

Indian railway is the largest public sector enterprise in the world. It caters to the transportation need of millions of people every day at low cost, maintenance and safety of railways as well as security of passengers have always been a herculean task ever since its inception (Rastogi, 2007). Suicide by collision with a train accounted for 1 to $12 \%$ of all suicides with up to $94 \%$ of all attempts resulting in death. Suicide by train seriously affects not only survivors but also train drivers and bystanders. Correlations between density of rail network, number of passengers and number of suicides by train have been found to be proportional (Krysinska and De Leo, 2008).

\section{CASE REPORT}

A 22 year old unmarried woman was found dead on the railway track along with a dead female newborn baby at 7:00 am on 6 August, 2010 (Figure 1). She was subjected for autopsy between $3: 45$ and $5: 15 \mathrm{pm}$ on the same day. Suicide note was brought by police (UDR no:133/2010 U/S 174 CrPC).
Autopsy

\section{External examination}

The following were noticed: left eye propped out of orbit; postmortem staining faintly present; rigor mortis setting in; dried blood stains; oil and grease stains present at places over the body; pregnancy changes; dead body of a female baby, part of placenta was brought along with the body.

\section{Injuries}

(1) Head crushed, front to back, skull shows multiple fractures, meninges lacerated irregularly, brain matter partially drained out, covered with blood and blood clots.

(2) Abdomen is crushed, $25 \times 20 \mathrm{~cm} \times$ abdominal cavity deep, extending from right flank to middle of left pelvis, with irregular laceration of liver, spleen and kidneys, coils of intestines protruded out, edges show contusion with grease stains (Figure 4).

(3) Crushed laceration, $22 \times 7 \mathrm{~cm} \times$ bone deep present over upper one third of right thigh with fracture of underlying femur bone.

(4) Crushed laceration, $8 \times 6 \mathrm{~cm} \times$ bone deep present over lower 


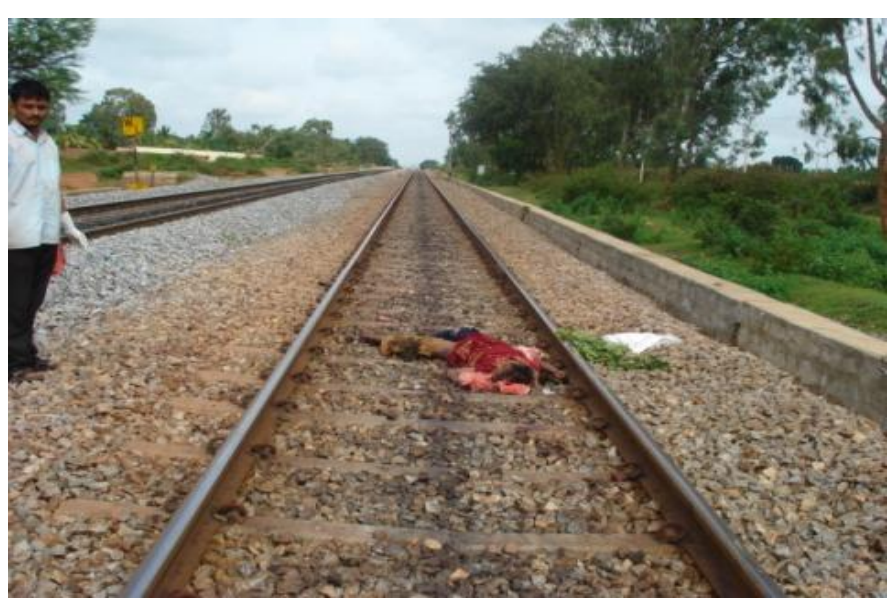

Figure 1. The scene where body was found.

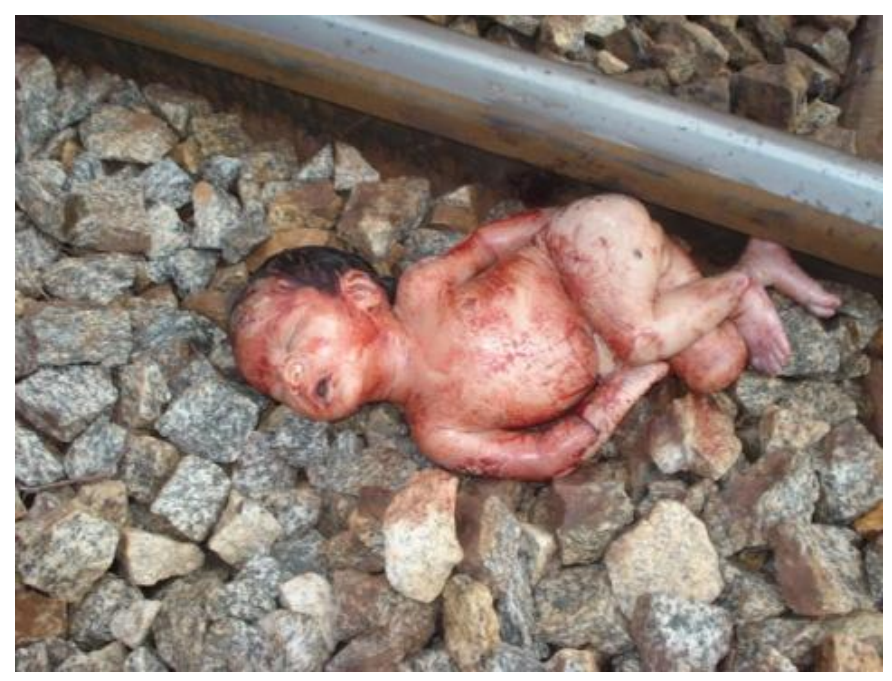

Figure 2. Newborn over rails.

one third of right leg with fracture of underlying bones.

(5) Crushed laceration, $8 \times 5 \mathrm{~cm} \times$ bone deep present over upper one third of left leg with fracture of underlying bones.

(6) Right shoulder joint, left wrist joint: fractured and dislocated.

(7) Second, third, and forth ribs on left side fractured at the sternal end with $100 \mathrm{ml}$ of blood in pleural cavity.

\section{Uterus}

Measures $18 \times 15 \times 3 \mathrm{~cm}$, weighs $583 \mathrm{~g}$ (gravid uterus) with irregular laceration, $15 \times 6 \mathrm{~cm} \times$ uterine cavity deep present over anterior surface of the fundus. Uterine walls were congested with remnants of torn membrane and placenta. The remaining part of placenta with torn cord was brought separately along with body (Figure 3).

\section{External genitalia}

No signs of stretching of vagina. Cervix is dilated upto $8 \mathrm{~cm}$ (Figure $5)$.

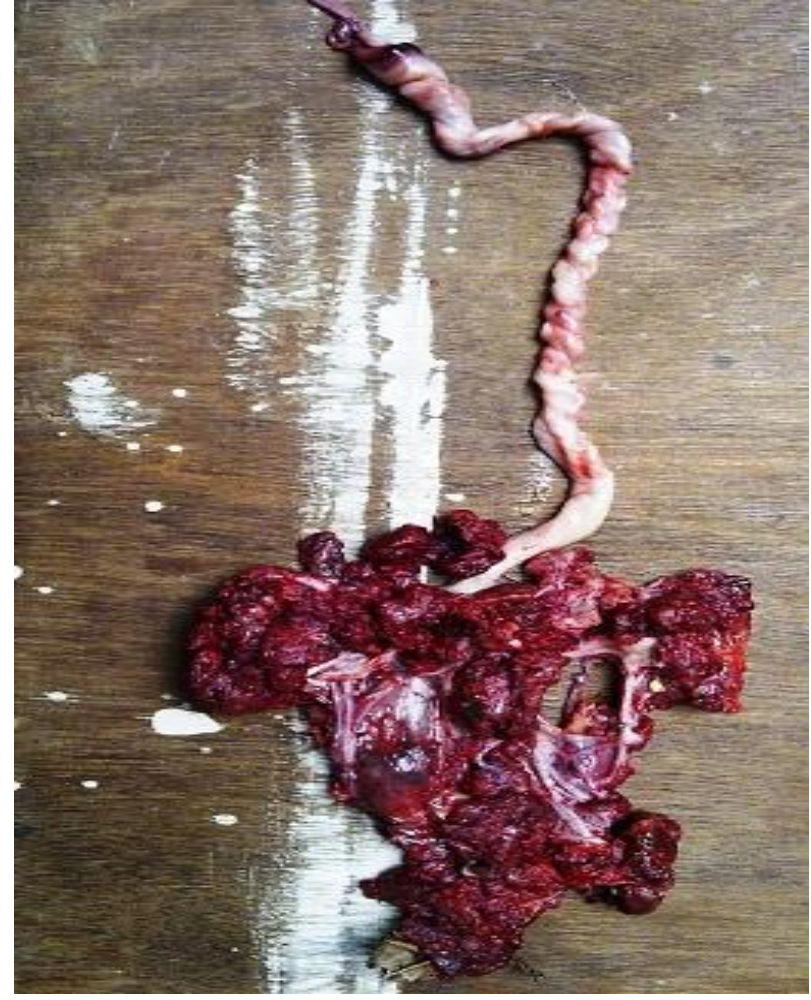

Figure 3. Lacerated placenta with cord.

\section{Examination of baby}

Dead body of female baby was brought along by police. Weighs $(3.2 \mathrm{~kg})$ measures $50 \mathrm{~cm}$ in length with signs of live birth (abdominal girth>chest, hydrostatic test positive, meconium present at rectum, ossification centre for lower end of femur and upper end of tibia appeared (Figure 2).

\section{Injuries on the baby}

Scalp laceration, $2 \times 3 \mathrm{~cm} \times$ bone deep, left side of forehead. Cranial vault shows comminuted fracture, meninges lacerated, brain covered by blood and blood clots. Spine C7 - T1 vertebra level, fractured (Figure 7).

\section{DISCUSSION}

The deceased, an adolescent unmarried pregnant female was found dead near rail track with fetus. The injuries mentioned earlier are consistent with train-pedestrain accident. Further, the history (suicidal note) and injuries over dead body points towards suicidal manner of death.

Regarding the manner of delivery, the injury over the abdomen and to the gravid uterus has led to the expulsion of fetus through the abdominal route. The uterus was large, the mucus membrane congested with remnants of torn placenta, these findings refute that uterus was gravid (Figure 6). 


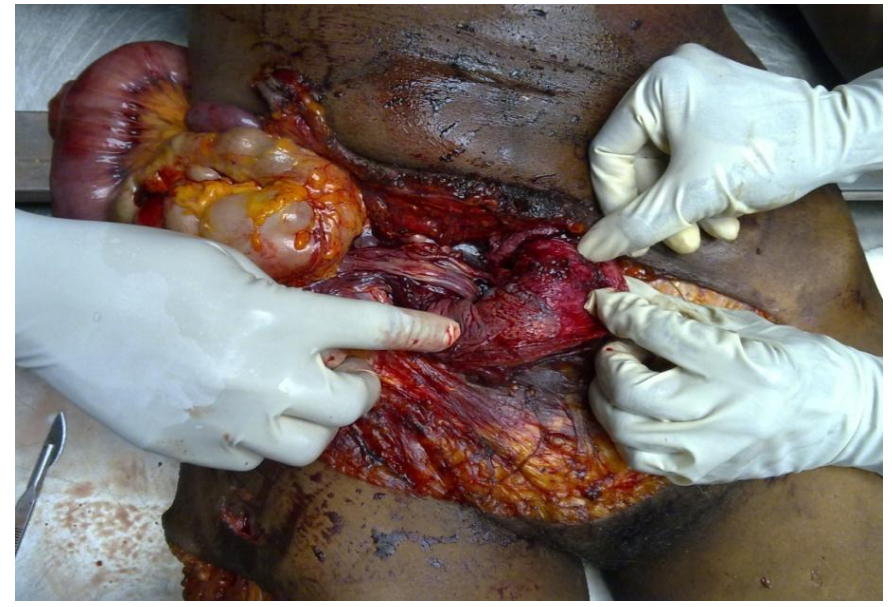

Figure 4. Laceration over abdomen exposing the lacerated uterus.

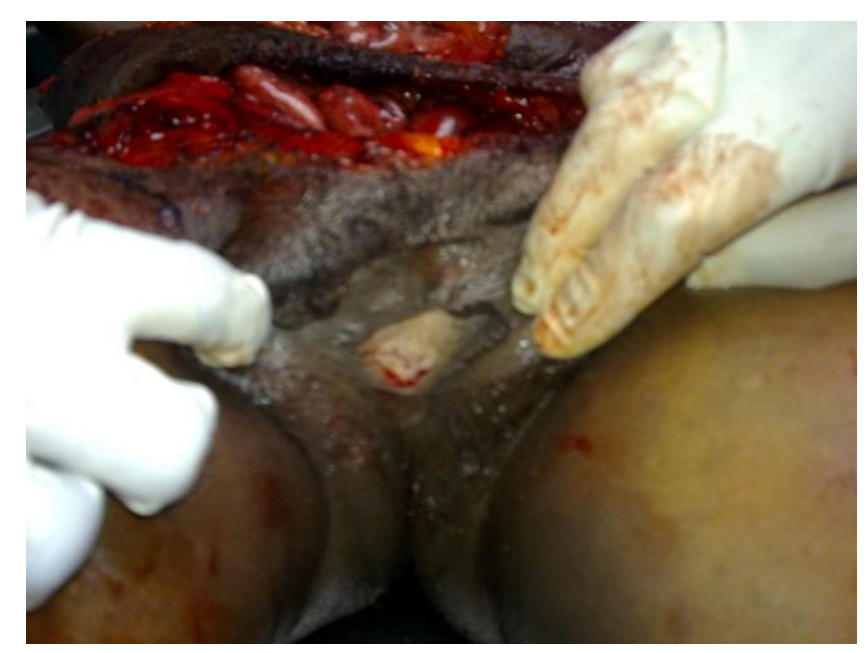

Figure 5. Per vaginal examination showing no signs of stretching of vagina with dilated cervix (upto $8 \mathrm{~cm}$ ).

Regarding whether she was in labour, she being a primigravida, her cervix appeared to have dilated up to 8 to $10 \mathrm{~cm}$ which occurs in the active phase of the 1st stage of labour and she could have been experiencing labour pains prior to death. However, there were no signs of vaginal stretching (per vaginal examination) which occurs in the 2nd stage during the expulsion of foetus through vagina. She had succumbed to the injuries by then after which the baby was expelled out of the injured uterus.

\section{Cause of death}

Death was due to multiple injuries sustained, consistent with railway injuries. Live born, full term female baby, expelled through the crushed abdomen and uterus, and the death was due to head injury sustained.

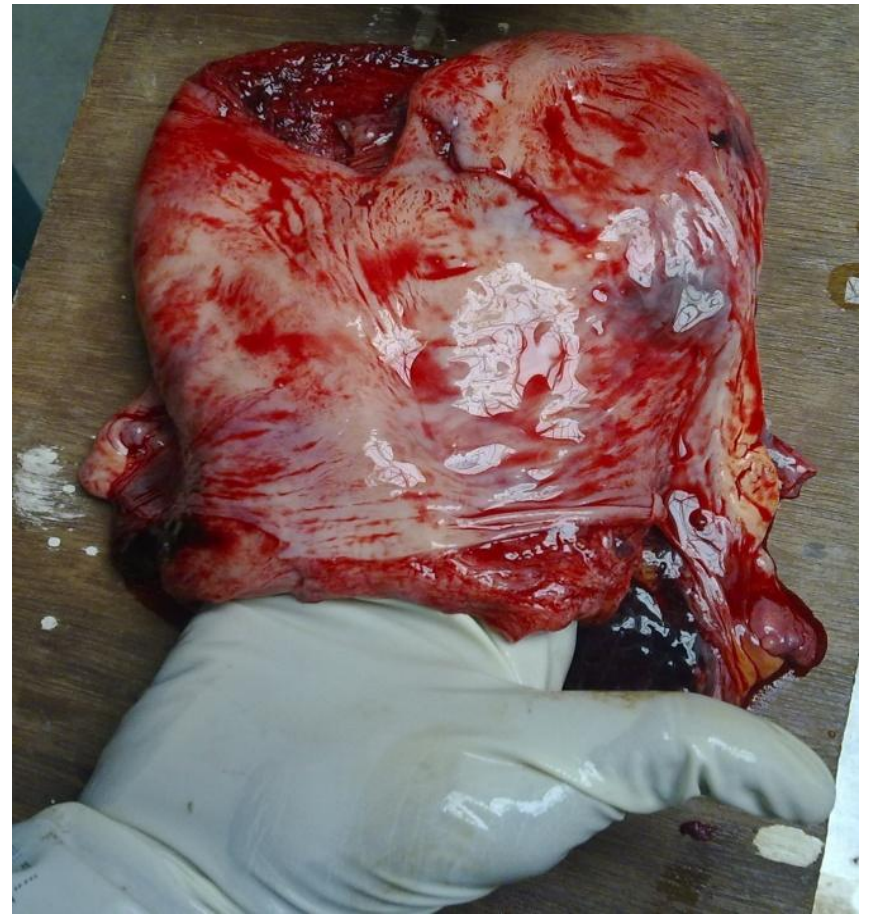

Figure 6. Laceration over the fundus of uterus and dilated cervix.

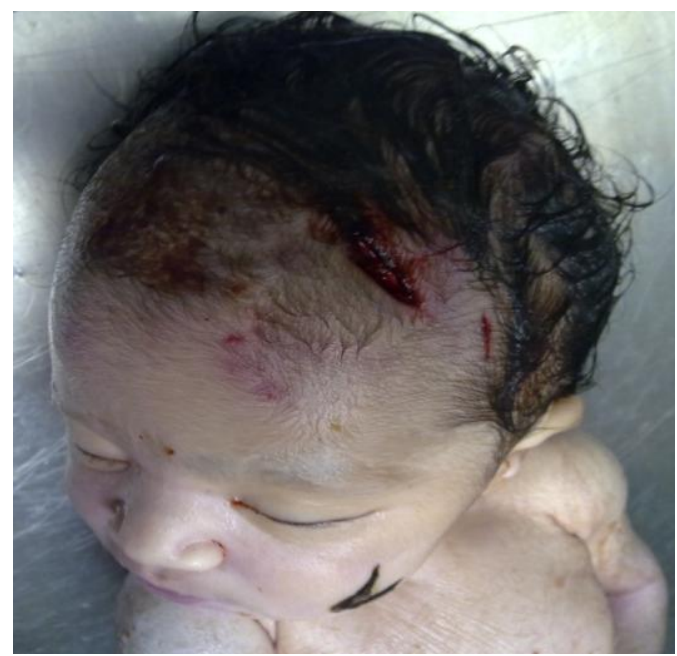

Figure 7. Head injury in the newborn.

\section{Suicide method}

Suicide method is any means by which one or more persons purposely kills themselves. Though individuals with suicidal feelings may consider the following methods most do not ultimately act upon them.

Some people commit suicide by deliberately placing themselves in the path of a large and fast-moving vehicle, resulting in a fatal impact. Some may throw themselves directly in front of an oncoming train or drive a car onto 
the tracks and sit inside while they wait for the train to arrive. People who commit suicide in this manner usually stay at or around the site. In suicides involving above ground railway lines, the victim will often simply stand or lie on the tracks waiting for the arrival of the train. As the trains usually travel at high speeds (usually between 80 and $200 \mathrm{~km} / \mathrm{h}$ ), the driver is usually unable to bring the train to a halt before the collision. This type of suicide may be traumatizing to the driver of the train and may lead to post-traumatic stress disorder. The sound of a train striking a person has been likened to that of hitting a pumpkin.

Suicide by being hit by a train has a $10 \%$ survival rate; a failed attempt typically results in severe injuries, including massive fractures, amputations and concussion, possibly leading to permanent brain damage and physical disability. Even when death occurs, it is not always painless and immediate.

Suicide methods can be classified according to two modes of interrupting life processes physically or chemically. Physical modes of interruption typically act by incapacitating the respiratory system or the central nervous system, usually by destruction of one or more key components. Chemical modes focus on interrupting biologically significant processes such as cellular respiration or diffusion capacity. Chemical methods of suicide produce latent evidence of action, whereas physical methods provide direct evidence.

Conflicting data exist on gender ratio of this type of suicide, but studies are homogenous in identifying young adults (20 to 40 year of age) as those most exposed to train suicide. Documented psychiatric diagnoses were found in up to $83 \%$ of cases. Mid-seasonal peaks were also identified, with events occurring mostly during late morning and early afternoon. Limited evidence exists for effective suicide prevention practices. Successful examples are represented by pits and sliding door systems (Singapore Mass Rapid Transit System) and responsible media reporting (Viennese Subway). Suicide by traininvolves emotionaland financial costs to individuals and society as a whole. A combination of different strategies might significantly reduce its effect (Krysinska and De Leo, 2008).

Postmortem examination study for data related to the age and sex of the victim, seasonal variations, type of train involved, part of the body affected and the pattern of injuries in different parts of the body was done. Detailed autopsy examination and subsequent statistical analysis was done (Pathak, 2009).

The suicidal events were evenly distributed by months and weekdays. However, most suicides occur during the day while unintentional events usually occur at night. Most train-person collisions happened in densely populated areas and $75 \%$ of the suicide victims were waiting on the track before the collision. Significance test between types of injury event (suicide, accident or unknown intent) showed small or no differences (Rådbo et al., 2005).
Close to $10 \%$ of all railway suicide attempts are non fatal. Fatality of suicidal behaviour on railway tracks is significantly associated with male sex pointing to a certain degree of ambivalence. Higher odds to die on open track area, fast track lines and during night-time suggest a reduced opportunity to survive due to circumstances (Erazo et al., 2005).

Most of the railway fatalities were accidental in nature and in the bread earning age group particularly among the males. The increasing number of population, overcrowding in the trains, reckless and careless behaviour of the passengers, pedestrians and the train drivers towards safety norms are the constant causes of railway fatalities.

People must follow some easy set of laws like do not travel on footboard, do not enter or get down from running trains, do not try to cross the level crossing gate when it is closed, be alert and reduce your speed while approaching railway unmanned level crossing. Never guess the speed of the train and adhere to the set norms of railway safety to curb this menace. The railway authority must take some steps to prevent the accidents by acknowledging the safety engineering, training and awareness among staff, attentive surveillance, high quality maintenance and strict law enforcement (Ramesh, 2010).

\section{Conclusion}

The high levels of the railway fatalities make a strong case for the necessary accident control interventions. Public as well as the railway authorities must take some measures to bring down these fatalities. Methods to reduce the rail-related suicides including CCTV surveillance of stretches where suicides frequently occur, with direct links to the local police or surveillance companies, which enables the police or guards to be on the scene within minutes after the trespassing was noted. Public access to the tracks should be made more difficult by erecting fences. Trees and bushes should be cut down around the tracks in order to increase driver visibility. Decrease the number of suicide attempts. Underground, deep drainage pit have the likelihood of fatality. Separation of the passengers from the track by means of a partition with sliding-doors is being introduced in stations, though it is expensive.

\section{REFERENCES}

Akhilesh P, Pankaj B, Mahajan AK, Bijaysingh R, Desai KP, Basu S (2009). Risking Limbs and Life - Railway fatalities in Vadodara: (A Retrospective Study), J. Forensic Med. Toxicol. 26(1): 54-57.

Erazo N, Baumert JJ, Ladwig KH J (2005). Affect Disord. Factors associated with failed and completed railway suicides, 88(2):137-43.

Krysinska K, De LD (2008). Suicide on railway networks: epidemiology, risk factors and prevention. Aust N Z J Psychiatry. 42(9):763-71.

Rådbo H, Svedung I, Andersson R (2005). Suicides and other fatalities from train-person collisions on Swedish railroads: a descriptive epidemiologic analysis as a basis for systems-oriented prevention. J. Safety Res. 36(5):423-8. 
Ramesh NW (2010). Analysis of Railway Fatalities in Central India, J Indian Acad Forensic Med. 32(4).311-314.

Rastogi P (2007). Railway accident - Complete decapitation. J. Punjab Academy Forensic Med. Toxicol. 7(1):35-37. 